\title{
Intervenciones basadas en mindfulness para reducir el estrés en la enfermedad cardiovascular: ¿cómo funcionan?
}

\author{
Mindfulness-based interventions for reducing stress \\ in cardiovascular disease: How do they work?
}

\author{
Erika Yohanna Bedoya Cardona ${ }^{1}$
}

Citación: Bedoya C., E.Y. (2019). Intervenciones basadas en mindfulness para reducir el estrés en la enfermedad cardiovascular: ¿cómo funcionan? Psicología y Salud, 29(1), 63-78.

\section{RESUMEN}

Las enfermedades cardiovasculares corresponden a un grupo de trastornos debidos al aumento del estrés, depresión, ansiedad, sedentarismo, consumo de tabaco y alcohol y otros factores, y encabezan la lista como causa de la mayor de mortalidad en países de ingresos medios y bajos. Las pérdidas atribuibles a dichas enfermedades aumentan la pobreza y constituyen uno de los mayores problemas de salud pública en el mundo. El objetivo de la presente revisión fue explicar teóricamente cómo funcionan las intervenciones basadas en el mindfulness para la regulación emocional y la reducción de estrés en estas afecciones, mediante una revisión bibliográfica que da cuenta de la investigación en Science Direct, PubMed, Ovid, Scielo, Psicodoc y Google Académico. En dichas fuentes se encuentra que el estrés y las enfermedades cardiovasculares se relacionan entre sí mediante mecanismos fisiológicos, psicológicos y conductuales. Se concluye que la implementación de las citadas intervenciones en la regulación emocional y la reducción del estrés puede ser efectiva debido a su fácil aprendizaje, el rol activo del paciente, su bajo costo, la posibilidad de llevarse a cabo en cualquier lugar y la ausencia de riesgos fisiológicos o psicológicos; sin embargo, aún no se establecen sus efectos sobre los parámetros fisiológicos, por lo que se requieren más estudios al respecto.

Palabras clave: Riesgo cardiovascular; Enfermedad cardiovascular; Estrés; Regulación emocional; Intervenciones basadas en mindfulness.

\begin{abstract}
Cardiovascular diseases involve disorders linked to increased stress, depression, anxiety, sedentary lifestyle, smoking, alcohol, etc., and lead the list of cause of mortality in low and middle income countries. Losses attributable to such illnesses increase poverty and are one of the greatest public health problems worldwide. The objective of the current review is to explain theoretically how mindfulness-based interventions work to the emotional regulation and stress reduction in cardiovascular diseases; on the basis of reviewing articles and books with research results in such databases as Science Direct, PubMed, Ovid, Scielo, Psicodoc and Google Scholar. This research literature shows that stress and cardiovascular diseases are related through physiological, psychological and behavioral mechanisms. It is concluded that the implementation of mindfulness-based interventions for emotional regulation and stress reduction in the treatment of cardiovascular diseases could be effective because they are easily learned, they involve the patient's active role, are low cost, they can be performed anywhere, and lack physiological or psychological risks. However, the effects of mindfulness on specific physiological parameters are not yet well established, and therefore further studies are required.
\end{abstract}

Key words: Cardiovascular risk; Cardiovascular disease; Stress; Emotional regulation; Mindfulness-based interventions.

\footnotetext{
${ }^{1}$ Facultad de Psicología, Universidad Cooperativa de Colombia, sede Bucaramanga, Bucaramanga, Santander, Colombia, tel. 57(76)85-45-00, correo electrónico: erika.bedoyac@campusucc.edu.co. Artículo recibido el 1 de septiembre de 2017 y aceptado el 16 de enero de 2018.
} 


\section{INTRODUCCIÓN}

$\mathrm{L}$ as enfermedades cardiovasculares (ECV en lo sucesivo) son un grupo de trastornos del corazón y los vasos sanguíneos que incluyen la cardiopatía coronaria, las enfermedades cerebrovasculares, las arteriopatías periféricas, las cardiopatías reumáticas y congénitas, las trombosis venosas profundas y las embolias pulmonares (Organización Mundial de la Salud [OMS], 2015), todas las cuales se relacionan con los cambios en el estilo de vida de la sociedad actual debidos al aumento del estrés, sedentarismo, consumo de tabaco, alcohol y sustancias psicoactivas, entre muchos otros (Ruiz, 2003). Este grupo de enfermedades encabezan la lista de mayor carga de mortalidad en países de ingresos medios y bajos (OMS, 2011). En 2008 se registraron 17 millones de muertes por ECV, lo que representa $30 \%$ del total en el orbe (Instituto Nacional de Salud y Observatorio Nacional de Salud, 2014). En 2011, 60 mil colombianos murieron a causa de las ECV, y actualmente el país enfrenta una epidemia de infartos, trombosis, hipertensión y diabetes, los cuales se hallan entre las diez principales causas de muerte (Ministerio de Salud y Protección Social de Colombia, 2014). Las pérdidas humanas y productivas atribuibles a las ECV representan dificultades económicas que aumentan la pobreza, disminuyen aún más la cobertura de los servicios para el tratamiento de estas afecciones y convierten a este tipo de enfermedades en uno de los problemas de salud pública con mayor relevancia en el mundo.

Diversos estudios epidemiológicos han demostrado que factores psicológicos como el estrés, la ansiedad o la depresión contribuyen a la instauración, manifestación y pronóstico negativo de la ECV (Dimsdale, 2008; Hamer, Molloy y Stamatakis, 2008; Richardson et al., 2012; Thurston, Rewak y Kubzansky, 2013). Respecto a la relación entre el estrés y la ECV, se ha desarrollado una hipótesis que sugiere que hay mecanismos fisiopatológicos y antecedentes comportamentales que son comunes en las dos entidades (Steptoe y Kivimäki, 2013). Los sistemas biológicos activados durante la respuesta al estrés son el eje hipotálamo-hipófisis-adrenal (cortical) y el eje simpático adrenal (medular). Cuando un estímulo estresante es percibido, se activan las neuronas hipofisiotróficas en el hipotálamo, secretando hormonas liberadoras que actúan en la hipófisis anterior y provocan la secreción de la hormona adrenocorticótropa, y luego cortisol en la glándula suprarrenal, lo que aumenta la movilización de la glucosa y los lípidos. Por otra parte, la percepción del estrés también activa neuronas simpáticas preganglionares, lo que se proyecta hasta los ganglios paravertebrales y a diferentes órganos. Los efectos de estos dos ejes están asociados con un aumento de la presión arterial, la hemostasis y la disfunción endotelial y la disminución de la sensibilidad a la insulina (Steptoe y Kivimäki, 2013).

El estrés, al igual que la aterogénesis, induce una respuesta inflamatoria con aumento de citoquinas (interleucina 6 y factor de necrosis tumoral alfa), estimula la activación plaquetaria y favorece cambios en las moléculas protrombóticas involucradas en la coagulación (Steptoe y Kivimäki, 2013); aumenta asimismo la frecuencia cardiaca, la tensión arterial, el tono y la reactivación vascular, los ácidos grasos libres, los triglicéridos y la respuesta inflamatoria. El estrés obliga al corazón a trabajar más intensamente. Las arterias coronarias que nutren al músculo cardíaco requieren un mayor aporte de energía. Además, la sangre se espesa y las arterias se vuelven menos reactivas o elásticas; se almacenan así sustancias nocivas en su pared, de forma que la sangre circula con mayor dificultad. A su vez, la fibrinólisis, que es el mecanismo defensivo que destruye los trombos, pierde efectividad. Igualmente, el exceso de respuesta simpática se asocia con trastornos en la conducción eléctrica del corazón, con una mayor vulnerabilidad a arritmias ventriculares $y$, por ende, con la muerte súbita (Arnau y Rodríguez, 2006).

Además de la reacción biológica al estrés, es necesaria una exposición continua a los eventos que activan dicha respuesta fisiológica (Steptoe y Kivimäki, 2013). Al respecto, un estudio de casos y controles con 15,152 pacientes con infarto agudo de miocardio (IAM) y 14,820 sin ECV encontró un odds ratio (OR) de 2 para el desarrollo de IAM en personas con alguna condición psicosocial adversa (Steptoe y Kivimäki, 2012). En relación a los eventos posteriores al alta hospitalaria tras haber tenido un IAM, se encontró que dentro de los primeros 30 días las rehospitalizaciones eran más 
comunes en pacientes con "alto estrés" durante la hospitalización respecto a aquellos con "bajo estrés" (Edmonson, Green, Ye, Halazun y Davidson, 2014).

Las respuestas al estrés surgen cuando los requerimientos para afrontar una condición exceden los recursos psicosociales o la capacidad de adaptación de las personas; es por esto que, dependiendo de la red de apoyo de cada individuo, así como de los rasgos de personalidad y el optimismo con el que se asimilan los cambios, los factores estresores son hechos subjetivos y varían según el caso (Witte, Bots, Hoes y Grobbee, 2000). Entre las situaciones más significativas consideradas como estresores crónicos en edades tempranas se encuentran el abuso sexual, el consumo de sustancias, las enfermedades de los padres y el bajo nivel socioeconómico (Steptoe y Kivimäki, 2013). Se ha encontrado un vínculo entre el estrés crónico debido al bajo nivel socioeconómico y el síndrome metabólico, un aumento del perímetro abdominal y del índice de masa corporal, dislipidemia, un incremento de los triglicéridos y bajos niveles de colesterol bueno (high-density lipoprotein, o HDL) (Drewnowski, 2009; Phillips y Klein, 2010). En un estudio prospectivo realizado a mujeres británicas, se encontró que las que presentaron alguna adversidad secundaria a su baja posición socioeconómica tenían una mayor mortalidad por ECV (1.4 veces más), en comparación con las que en su niñez habían tenido una posición socioeconómica alta (Power, Hypponen y Smith, 2005).

Otro factor que guarda una estrecha relación con la ECV es la ansiedad (Roest, Martens, de Jonge y Denoller, 2010). La prevalencia de síntomas ansiosos en pacientes con enfermedad cardiaca crónica va de 5 a $10 \%$, la cual se eleva a $50 \%$ en pacientes con enfermedad cardiaca aguda en la unidad de cuidados intensivos. La asociación entre ansiedad y ECV puede explicarse por la evidencia que existe de que las emociones negativas son un factor potencial de riesgo (Kubzansky y Kawachi, 2000). Strik, Denollet, Lousberg y Honig (2003) aplicaron pruebas de ansiedad, depresión y hostilidad a 318 sujetos con una media de edad de 58 años, sobrevivientes a un primer ataque cardíaco. Después de un seguimiento de 3.4 años, hubo 25 eventos cardíacos fatales y no fatales. Los sínto- mas de ansiedad estaban asociados a eventos cardíacos y al uso de antidepresivos. La ansiedad fue un predictor independiente de ambos eventos. Los investigadores concluyeron que los síntomas de ansiedad necesitan considerarse en el riesgo y el posterior tratamiento de pacientes después de sufrir un IAM.

En un estudio con 288 pacientes hospitalizados por IAM, se encontró que $26 \%$ de ellos sufría altos niveles de síntomas ansiosos (Lane, Carroll, Ring, Beevers y Lip, 2001). Por otra parte, Albert, Chae, Rexrode, Manson y Kawachi (2005) examinaron la relación entre la ansiedad fóbica y la enfermedad cardíaca coronaria aplicando el índice de Crown-Crispque (CCI) a 72,359 mujeres sin historial de ECV o de cáncer en el año 1988. En los doce años siguientes se produjeron 96 muertes súbitas cardíacas, 930 IAM no mortales y 267 muertes por ECV. Se explicaron dichos resultados por la tensión, misma que fue observada como un factor de riesgo independiente en incidentes de la enfermedad. Eaker, Sullivan, Kelly-Hayes, D’Agostino y Benjamin (2005) examinaron a 3,682 mujeres, con una media de edad de 48.5, y siguieron a lo largo de diez años la incidencia de la enfermedad y la mortalidad; para ello, recogieron medidas de tensión, ansiedad y factores de riesgo. En sus resultados hallaron diferentes síntomas producidos por la ansiedad, como sensaciones desagradables (temor, preocupación, tensión, inseguridad, etc.), acompañadas de sentimientos y pensamientos negativos sobre sí mismas. Además, esta experiencia estuvo unida a una alta activación fisiológica (especialmente del sistema nervioso autónomo y del sistema somático), así como a una actividad poco ajustada y desorganizada del comportamiento.

Dichos resultados pueden deberse a que el estrés y la ansiedad crónica producen taquicardia ventricular, lo que aumenta las muertes súbitas por IAM (Roest, Martens, Denollet y de Jonge, 2010). El mecanismo funciona de una manera tal que el estrés predispone a que el hipotálamo aumente la liberación de corticotropina, estimulando con ello el que la pituitaria anterior libere una mayor cantidad, lo que a su vez estimula a la glándula suprarrenal para que produzca más cortisol, que ha sido asociado al desarrollo de aterosclerosis e hipertensión arterial. Además, en pacientes ansiosos se produce una hiperactividad del eje hipotalámi- 
co-hipofisario-adrenal y del sistema simpáticoadrenal, vinculada a la aparición de aterosclerosis, incremento de la presión sanguínea y del índice de viscosidad sanguínea, formación de émbolos y aumento de la resistencia vascular periférica (Alarcón y Ramírez, 2006).

Por otra parte, la depresión aparece en uno de cada cinco pacientes en las semanas posteriores a haber sufrido un IAM (Nicholson, Kuper y Hemingway, 2006). Lo anterior se considera un indicador de mal pronóstico al asociarse a la recurrencia de eventos coronarios y de mortalidad. En un estudio del Centro Médico Universitario de Duke (Estados Unidos), en el que participaron 357 pacientes durante un período de quince meses (35.3\% con síntomas de depresión y $13.9 \%$ con depresión grave), se encontró que los pacientes con falla cardiaca y sin depresión tuvieron una mortalidad de $5.7 \%$ a los tres meses y de $13.7 \%$ transcurrido un año, mientras que la mortalidad de los pacientes con falla cardiaca y con depresión aumentó de $13 \%$ a los tres meses a $16.1 \%$ al año. Ante estos resultados, los autores concluyeron que una posible explicación de los mismos es que los síntomas depresivos facilitan estilos de vida poco saludables, como el sedentarismo, el tabaquismo y la falta de adherencia a los tratamientos o el incumplimiento de las prescripciones médicas (Durham, 2001).

Hay evidencia de algunos mecanismos conductuales y fisiológicos que podrían explicar la relación entre la depresión y las ECV: 1) La cardiotoxicidad de los antidepresivos (Carney, Freedland, Miller y Jaffe, 2002; Cohen, Gibson y Alderman, 2000); 2) Los factores de riesgo como el tabaquismo (Messner y Bernhard, 2014; Villablanca, McDonald y Rutledge, 2000), la hipertensión (Joynt, Whellan y O'Connor, 2003), la diabetes (Carney et al., 2002; Clouse, Freedland, Carney, Griffith y Lustman, 2001; Kimberly et al., 2000), el colesterol (Angheel, Ghiuru y Gavrilescu, 2011) y el sedentarismo (Khawaja, Westermeyer, Gajwani y Feinstein, 2009; Lampinen, Heikkinen y Ruoppila, 2000); 3) La no adherencia a los tratamientos y a la prevención (DiMatteo, Lepper y Croghan, 2000; Ziegelstein et al., 2000), y 4) la baja variabilidad de la frecuencia cardiaca, responsable de un tono cardiaco autonómico alterado (García-Gómez, Lopez-Jaramillo y Tomaz, 2007), el incremento en la agregación plaquetaria (Musselman et al., 2000; Pollock, Laghrissi-Thode y Wagner, 2000) y los procesos inflamatorios (Papaioannou, Pneumatikos y Maglaveras, 2013).

Según los datos reportados, es necesaria la puesta en práctica de estrategias que logren modificar las respuestas fisiológicas y emocionales ante sucesos estresantes, el fortalecimiento de los recursos de adaptación y afrontamiento de problemas y la implementación de un entrenamiento en técnicas de regulación emocional que hagan posible reducir el impacto negativo de estos factores psicológicos sobre la ECV. Los estudios al respecto han sido escasos, con poca calidad metodológica, tamaños muestrales pequeños y corta duración del seguimiento de los resultados. Además, la comprensión de los mecanismos y los marcos teóricos mediante los cuales las intervenciones influyen sobre las ECV también han sido poco explorados. El objetivo de la presente revisión fue, por consiguiente, explicar teóricamente cómo funcionan las intervenciones basadas en el mindfulness ("atención plena") para la regulación emocional y la reducción del estrés, como un complemento a los diversos tratamientos médicos o quirúrgicos en personas con ECV, mediante la revisión bibliográfica de artículos y libros que reportan los resultados de la investigación, para lo cual se buscaron los términos "estrés", “ansiedad", "depresión”, “enfermedad cardiovascular" y "mindfulness" en las bases de datos Science Direct, PubMed, Ovid, Scielo, Psicodoc y Google Académico.

\section{Terapias de reducción del estrés}

Teniendo en cuenta los antecedentes señalados, si bien las opciones de tratamiento para las ECV han tenido un crecimiento importante, sus resultados no son definitivos pues solamente alivian los síntomas y disminuyen las posibles complicaciones, lo que sin embargo resalta la importancia de reducir los factores de riesgo y establecer los cambios necesarios en el estilo de vida en el largo plazo con el fin de modificar el curso de la enfermedad, disminuyendo así su progreso y el riesgo de muerte súbita (Naranjo, Díaz y García, 2012). Aunque son muchas las publicaciones sobre la rehabilitación cardiaca, aún no se ha definido un método que sea totalmente eficaz para la reducción defini- 
tiva de las recurrencias, reingresos hospitalarios y mortalidad (Balady et al., 2007). En una revisión publicada por Jolliffe et al. (2001) se encontró una reducción de mortalidad en doce estudios sobre terapia basada en ejercicios únicamente. A pesar de tales resultados benéficos, todavía hay barreras que impiden la adherencia a este tipo de terapias, lo que disminuye su efectividad (Giallauria et al., 2009). Algunos estudios recientes han mostrado que la inclusión del manejo de factores psicológicos en los programas de rehabilitación tradicionales puede tener un efecto benéfico adicional al reducir el riesgo. Ciertas terapias complementarias y prácticas de reducción de estrés contribuyen al mejoramiento de pacientes con ECV (Leonaite y Vainoras, 2010; Neves, Alves, Ribeiro, Lopes y Oliveira, 2009; Yeh, Davis y Phillips, 2006; Younge, Gotink, Baena, Roos-Hesselink y Hunink, 2014). Una extensa revisión de intervenciones psicosociales (van Dixhoorn y White, 2005), tales como manejo del estrés (Ades, Balady y Berra, 2001; Blumenthal, Sherwood y Babyak, 2005), terapia cognitivo-conductual (Cully et al., 2010; Dekker, 2011), relajación (Castillo-Richmond et al., 2000; Emani y Binkley, 2010), educación para la salud (Chaves et al., 2015; Hayman et al., 2004), imaginería (Varvogli y Darviri, 2011), hipnosis (Vickers, Zollman y Payne, 2001) y yoga (Manchada y Madan, 2014) han demostrado ser bastante efectivas para reducir la angustia, la tensión arterial, los niveles de colesterol, la recurrencia cardiaca y la mortalidad (Arthur, Patterson y Stone, 2005; Kreitzer y Snyder, 2002).

La terapia de relajación se define como el entrenamiento para inducir una reducción de tensión por el mismo paciente (Delui, Yari, Khouyinezhad, Amini y Bayazi, 2013; Jacobs, 2001). La mayoría de las técnicas se centran en la atención (activa o pasiva), en representaciones mentales, imágenes, palabras, pequeños movimientos o cambio de postura, contracción y relajación muscular, respiración o biorretroalimentación, entre otros. Dichas técnicas han mostrado que los pacientes muestran una importante recuperación después de un evento isquémico cardíaco mediante la reducción de la ansiedad, la frecuencia cardíaca, la angina de pecho y el riesgo de muerte, así como un aumento de la reinserción laboral (Day, 2000; van Dixhoorn y White, 2005). Por su parte, la téc- nica de relajación muscular progresiva (RMP) ha demostrado tener beneficios fisiológicos y psicológicos, como una baja percepción del estrés (Dehdari, Heidarnia, Ramezankhani, Sadeghian y Ghofranipour, 2009; Kabat-Zinn, 2003; Sheila, Irvin, Lin y Mar, 2003).

Investigaciones previas sobre la reducción de estrés mediante la técnica de meditación trascendental (MT) han reportado mejorías frente a los factores de riesgo de ECV (Walton et al., 2002), reduciendo significativamente el riesgo de IAM, accidentes cerebrovasculares y mortalidad, lo que la hace una práctica clínicamente útil en la prevención secundaria (Schneider et al., 2012). Durante la práctica de MT ocurre una reducción en la actividad mental y física como resultado de un estado llamado "conciencia trascendental" (Walton, Schneider y Nidich, 2004), al cual se le atribuye la restauración del funcionamiento normal de varios sistemas, como el nervioso autónomo, el neuroendocrino, el cardiovascular y el inmune (McEwen, 2000), todos ellos involucrados en la adaptación a ambientes estresantes o desafiantes (Walton et al., 2004). Por tanto, se sugiere que la reducción de ECV que ocurre gracias a la técnica MT estaría más relacionada con un bajo estrés psicosocial y con la adecuada reducción de sus efectos (Pace et al., 2009).

\section{Intervenciones basadas en mindfulness}

Otra herramienta prometedora son las intervenciones basadas en mindfulness (IBM), las cuales, por medio de la meditación, permiten mantener la atención en el momento presente, de una forma abierta y sin prejuicios (Vallejo, 2006), sin atender situaciones del pasado o el futuro. Esta intervención ayuda a evitar pensamientos automáticos y hábitos y patrones de conducta poco saludables, como preocupación, miedo, enojo y evitación (Hayes y Feldman, 2004; Kabat-Zinn, 2013; Keng, Smoski y Robins, 2011), aumentando así la posibilidad de una regulación que favorece el bienestar físico y psicológico (Chiesa y Serretti, 2009, 2010; Hervás, Cebolla y Soler, 2016; Mars y Abbey, 2010). En las prácticas formales se entrena a la persona en habilidades para sostener la atención en el momento presente, el cuerpo, las emociones, los pensamientos y las sensaciones, lo que genera una 
mayor conciencia de los mismos. Las prácticas informales se enfocan en acontecimientos de la vida cotidiana, en las situaciones agradables y desagradables, teniendo como herramienta fundamental la respiración, las comunicaciones interpersonales y las actividades rutinarias, como comer, conducir o caminar. Aunque la duración de la intervención es breve, busca fomentar la autorregulación, la autosuficiencia y el aprendizaje permanente de un método que puede mejorar la salud y el bienestar durante toda la vida (Santorelli, 2014).

Usualmente, las IBM se agrupan como programas, siendo los siguientes los más reconocidos, estandarizados y aplicados a diferentes tipos de población (Abbott et al., 2014): la reducción del estrés (MBSR) (Kabat-Zinn et al., 1992) y la terapia cognitiva (MBCT) (Segal, Williams y Teasdale, 2002) basadas en mindfulness, la terapia de aceptación y compromiso (ACT) (Hayes, Strosahl y Wilson, 1999), la terapia de conducta dialéctica (DBT) (Linehan, 1993) y la prevención de recaídas (MBRP) (Marlatt y Donovan, 2005).

\section{Entendiendo el mindfulness y su funcionamiento en las ECV}

El concepto de mindfulness tiene sus raíces en el budismo y otras tradiciones relativas a la atención consciente y la conciencia activa, las cuales se orientan de manera abierta y receptiva a la experiencia presente (Brown y Ryan, 2003), pero se diferencian de aquella atención que puede ser dividida, debilitada o restringida por la rumiación, la inmersión en el pasado, las fantasías o ansiedades sobre el futuro que pueden ser motivadas defensivamente, llevando así al rechazo para reconocer o atender pensamientos, emociones u objetos de percepción, así como a actuar de manera compulsiva o automática (Ryan y Deci, 2000).

Según la teoría de autodeterminación (self determination theory), o SDT (Ryan y Deci, 2000), la conciencia abierta facilita la elección de conductas más consistentes con las propias necesidades, valores e intereses. El mindfulness facilita el bienestar a través de actividades autorreguladas que respondan a tales necesidades con autonomía, competencia y congruencia (Hodgins y Knee, 2002). Considerando entonces el mindfulness como la aten- ción plena y sostenida sobre la experiencia actual sin juzgarla, Brown y Cordon (2009) argumentan que ésta debe tener un contenido emocional y unos correlatos regulatorios característicos que podrían explicarse por tres razones: 1) Debido a que implica una desconexión del procesamiento conceptual habitualmente evaluativo, debería conducir a estados afectivos más equilibrados y menos displacenteros (Brown y Ryan, 2003; Varela y Depraz, 2003); 2) Con una percepción más clara y objetiva, los eventos y experiencias potencialmente desafiantes tendrían menos probabilidad de ser distorsionados por sesgos cognitivos o malinterpretaciones que puedan generar respuestas emocionales desagradables (Brown, Ryan y Creswell, 2007), y 3) La calidad de la atención influye en los resultados regulatorios de la emoción, y puesto que el mindfulness favorece una atención sostenida y abierta a los fenómenos internos y externos tal y como son, podría disuadir la tendencia a usar reguladores desadaptativos como rumiación y supresión del pensamiento, y por el contrario podría estimular la exposición voluntaria a eventos desagradables para promover una regulación emocional más adaptativa (Feldner, Zvolensky, Eifert y Spira, 2003; Gross y Thompson, 2007; Levitt, Brown, Orsillo y Barlow, 2004; Sloan, 2004).

Diversos estudios empíricos evidencian que el mindfulness, como rasgo y como práctica meditativa, se asocia con una menor supresión de pensamientos, rumiación, impulsividad, pasividad y otras formas de regulación desadaptativa relacionadas con una pobre salud mental (Baer, Smith, Hopkins, Krietemeyer y Toney, 2006; Brown y Ryan, 2003; Cardaciotto, Herbert, Forman, Moitra y Farrow, 2008; Chambers, Lo y Allen, 2008; Feldman, Hayes, Kumar, Greeson y Laurenceau, 2007; Frewen, Evans, Maraj, Dozois y Partridge, 2008; McKee, Zvolensky, Solomon, Bernstein y Leen-Feldner, 2007; Shapiro, Brown y Biegel, 2007; Wupperman, Neumann y Axelrod, 2008). Por otra parte, las habilidades en el mindfulness se asocian positivamente con estrategias reguladoras adaptativas, como la aceptación de pensamientos negativos (Baer et al., 2006; Brown y Ryan, 2003; Frewen et al., 2008), la expresión de emociones sin alterar su trayectoria (Barrett y Gross, 2001), y la satisfacción y una mayor capacidad para respon- 
der al estrés (Barnes, Brown, Krusemark, Campbell y Rogge, 2007; Wachs y Cordova, 2007). Dichas habilidades se pueden establecer mediante una estrategia reguladora entendida como capacidad de afrontamiento (Gross y Thompson, 2007; Larsen, 2000), que se presenta en tres formas: afrontamiento activo de la situación estresante, aceptación de la realidad y reinterpretación cognitiva, cuyo fin es facilitar la asimilación y aumentar el bienestar (Fortune, Richards, Main y Griffiths, 2002; Lyne y Roger, 2000; Stowell, Kiecolt-Glaser y Glaser, 2001).

En un estudio realizado por Arch y Craske (2006), en el cual se compararon un grupo con entrenamiento en ejercicios de respiración para inducir un estado mindfulness con otro grupo entrenado en desenfocar la atención y la preocupación, se encontró que aquél mostró menos reactividad negativa, menos volatilidad emocional y más disposición para mantener contacto con fotos de imágenes aversivas, hallazgo que sugiere que la IBM genera efectos saludables en la regulación emocional y la salud mental. Por otra parte, Creswell, Way, Eisenberger y Lieberman (2007) evaluaron el beneficio protector de la atención mindfulness ante la amenaza de exclusión social, hallando mediante imágenes cerebrales una reactividad reducida en las regiones implicadas en la experiencia de la ansiedad social y el dolor, evidenciando así que el mindfulness puede disminuir la reactividad y la evitación en situaciones sociales amenazantes y angustiantes (Allen y Knight, 2005).

En otro estudio realizado por Bostock, Hamer, Wawrzyniak, Mitchell y Steptoe (2011) se encontró que personas con un estilo emocional positivo, caracterizado por bienestar, vigor y calma, percibían una tarea estresante como menos difícil, y a sí mismos con un mayor grado de control. Kabat-Zinn et al. (1992), mediante un programa de ocho semanas de MBSR, también demostraron una reducción efectiva de los síntomas de ansiedad y dolor (Kabat-Zinn, Lipworth y Burney, 1985). Asimismo, varios estudios que emplearon dicho programa han demostrado resultados positivos en muestras médicas y psiquiátricas de adultos y de estudiantes (Langer, Ulloa, Cangas, Rojas y Krause, 2015; Linehan, Cochran y Kehrer, 2001; Miró et al., 2011; Reibel, Greeson, Brainard y Rosenzweig,
2001; Speca, Carlson, Goodey y Angen, 2000; Teasdale et al., 2000; Williams, Kolar, Reger y Pearson, 2001).

Recientemente se ha propuesto que las IBM son una estrategia efectiva para reducir las complicaciones y mejorar la funcionalidad de pacientes con padecimientos físicos (Ludwing y Kabat-Zinn, 2008; Proulx, 2008; Sánchez y Moreno, 2017; Teasdale et al., 2000; Wansink, Painter y North, 2005), enfermedad crónica (Bohlmeijer, Prenger, Taal y Cuijpers, 2010) y ECV (Lawrence, Booth, Mercer y Crawford, 2013; Sullivan et al., 2009). Si bien los estudios sobre mindfulness en ECV son relativamente pocos (Loucks et al., 2015), algunos han demostrado su eficacia para reducir la presión arterial (Blom et al., 2014; De la Fuente, Franco y Salvador, 2010; Hughes et al., 2013) y la fatiga mental en pacientes con infarto y trauma cráneoencefálico (Johansson, Bjuhr y Ronnback, 2012). A su vez, Tacón, Caldera y Ronaghan (2004) mostraron que al aplicar una IBM a mujeres con algún tipo de ECV, se redujo su puntaje en una escala de expresión del afecto negativo previo a la intervención. Edelman et al. (2006) plantearon en su estudio que un plan multifactorial basado en herramientas personalizadas y creativas reduce el riesgo de $\mathrm{ECV}$, especialmente porque lograron aumentar la disposición a llevar a cabo actividades físicas y los días de ejercicio (2.4 vs. 3.7) y disminuir el peso corporal. La intervención tuvo como resultado una disminución de $16 \%$ en la escala de Framingham en este grupo, en comparación con solo $12 \%$ en el grupo control. Otro estudio de Nyklíček, Dijksman, Lenders, Fonteijn y Koolen (2014) muestra que las IBM disminuyen el estrés psicológico y psicosocial y mejoran la calidad de vida de los pacientes con ECV sometidos a intervención coronaria percutánea, cuando se les comparó con pacientes a los que solamente se les dio una terapia de autoayuda.

Respecto a la eficacia de las IBM sobre los factores de riesgo de ECV, se ha encontrado una influencia potencial de las mismas en los niveles de actividad física (Loucks, Britton, Howe, Eaton y Buka, 2015; Salmoirago-Blotcher, Husinger, Morgan, Fischer y Carmody, 2013; Ulmer, Stetson y Salmon, 2010), en términos de que las personas muestran una mejor disposición para estar físi- 
camente activas (Ivanova, Jensen, Cassoff, Gu y Knauper, 2015; Kangasniemi, Lappalainen, Kankaanpaa, Tolvanen y Tammelin, 2015; Moffitt y Mohr, 2015). El entrenamiento mindfulness para dejar de fumar (De Souza et al., 2015) ha demostrado ser más eficaz que los programas de abstinencia (Brewer et al., 2011; Davis et al., 2014). En cuanto a la dieta, se han visto sus resultados en la reducción de la ingesta calórica (Miller, Kristeller, Headings y Nagaraja, 2014), el aumento en el consumo de frutas y verduras y una reducción en el de postres y dulces (Ouwens, Schiffer, Visser, Raeijmaekers y Nyklicek, 2015), lo cual se relaciona con un comportamiento alimenticio emocional más infrecuente (Kearney et al., 2012; Tak et al., 2015), que a su vez conlleva la pérdida de peso en pacientes obsesos (Olson y Emery, 2015; O'Rei1ly, Cook, Spruijt-Metz y Black, 2014). Cuando se practica la terapia en grupos, existe el beneficio adicional de aumentar el apoyo social, contrarrestando así el aislamiento que tanto se ha relacionado con las ECV (Steptoe, Shankar, Demakakos y Wardle, 2013; Thurston y Kubzansky, 2009).

Los mecanismos mediante los cuales se podría explicar cómo funcionan las IBM sobre dichos factores de riesgo consiste en que, al tener un mayor control atencional de las emociones y sensaciones negativas y la ansiedad (Mehling et al., 2009) -que conducen, por ejemplo, al consumo de tabaco o de alimentos grasos o hipercalóricos, y al sedentarismo, entre otros-, la persona puede ver con más claridad la disonancia, reconocer y delimitar lo que es verdaderamente significativo o valioso, y aumentar su motivación intrínseca para disminuir la conducta de riesgo (Shapiro, Carlson, Astin y Freedman, 2006) en virtud de la conciencia de la manera en que responde al estrés (Kabat-Zinn, 2013), tratando las emociones y sensaciones como eventos pasajeros (Elwafi, Witkiewitz, Mallik, Iv y Brewer, 2013) y acrecentando su sentido de autoconciencia, autoeficacia y control (Jerant, Moore, Lorig y Franks, 2008; Kelly y Greene, 2014) a través de los siguientes tres componentes principales del mindfulness expuestos en la teoría propuesta por Shapiro et al. (2006): descentramiento (salir de la experiencia inmediata), desautomatización (deshacer el proceso automático que controla la percepción y cognición) y desprendimiento (adoptar una actitud fenomenológica), mediante los cuales la persona puede reducir sus cogniciones negativas y patrones de pensamiento automáticos (Yeung, 2013).

\section{CONCLUSIÓN}

De acuerdo con Steptoe y Kivimaki (2013), el estrés y las ECV se relacionan mediante mecanismos fisiológicos, psicológicos y conductuales que ocurren de manera similar en ambas entidades; es decir, los cambios fisiológicos y la activación de sistemas que ocurren en la respuesta al estrés influyen de manera importante en el desarrollo de la ECV cuando se producen de manera repetida a lo largo del tiempo. Aunque el estrés puede influir a través de diversas vías sobre la salud cardiovascular, una vía importante por la cual puede perturbar la salud del corazón es un incremento de las emociones negativas, especialmente la hostilidad, la ansiedad y la depresión, que son las variables emocionales negativas que de forma más consistente han sido asociadas en los últimos años a los problemas cardiovasculares (Sandín, 2001).

Los estudios sobre IBM aplicadas específicamente a pacientes con ECV muestran mejorías en la percepción de la salud, la calidad de vida y las respuestas psicológicas (Abbott et al., 2014; Hartmann et al., 2012; Joo, Lee, Chung y Shin, 2010; Keen, Duncan y Gold, 2012; Moustgaard, Bedard y Felteau, 2007; Nyklíček et al., 2014; Rosenzweig et al., 2007; van Son et al., 2013). Los resultados de las investigaciones previamente mencionadas sugieren entonces que el mindfulness se asocia con indicadores afectivos y cognitivos de bienestar (Kabat-Zinn, 2013; Shapiro, Schwartz y Bonner, 1998), posibilitando así que las personas reaccionen menos intensamente ante los sentimientos negativos y amenazantes derivados de las experiencias desagradables, y recuperen más rápidamente sus niveles fisiológico y psicológico (Brown y Cordon, 2009; Frewen et al., 2008; Kiken y Shook, 2011).

La implementación de IBM en la regulación emocional y la reducción del estrés en el tratamiento de las ECV puede resultar bastante efectiva debido a su fácil aprendizaje, pues el paciente asume un rol más activo dentro del tratamiento; son además de bajo costo, pueden llevarse a cabo en casi cualquier lugar, y no representan riesgos fisiológicos o psicológicos (Younge et al., 2014); 
sin embargo, aún no se establecen de manera clara, definitiva ni unánime sus efectos en los parámetros fisiológicos de la enfermedad (Abbot et al.,
2014), y por tanto se requieren más estudios al respecto.

\section{REFERENCIAS}

Abbott, R.A., Whear, R., Rodgers, L.R., Bethel, A., Thompson Coon, J., Kuyken, W. y Dickens, C. (2014). Effectiveness of mindfulness-based stress reduction and mindfulness based cognitive therapy in vascular disease: A systematic review and meta-analysis of randomised controlled trials. Journal of Psychosomatic Research, 76, 341-351.

Ades, P.A., Balady, G.J. y Berra, K. (2001). Transforming exercise-based cardiac rehabilitation programs into secondary prevention centers: a national imperative. Journal of Cardiopulmonary Rehabilitation and Prevention, 21, 263-272.

Alarcón, R. y Ramírez, E. (2006). Medicina psicosomática en enfermedad cardiovascular: Consideraciones clínicas. Revista Colombiana de Psiquiatría, 14(19), 112-124.

Albert, C., Chae, C., Rexrode, K., Manson, J. y Kawachi, I. (2005). Phobic anxiety and risk of coronary heart disease and sudden cardiac death among women. Circulation, 111(4), 480-487.

Allen, N.B. y Knight, W.E.J. (2005). Mindfulness, compassion for self and compassion for others: Implications for understanding the psychopathology and treatment of depression. En P. Gilbert (Ed.): Compassion: conceptualisations, research and use in psychotherapy (pp. 239-262). London: Routledge.

Angheel, L., Ghiuru, R. y Gavrilescu, C.M. (2011). Depression and cardiovascular diseases in the elderly. Revista Medico-Chirurgicala a Societatii De Medici Si Naturalisti Din Iasi, 115(2), 331-336.

Arch, J.J. y Craske, M.G. (2006). Mechanisms of mindfulness: Emotion regulation following a focused breathing induction. Behavoiur Research and Therapy, 44, 1849-1858.

Arnau, M. y Rodríguez, M. (2006). Estrés y enfermedad cardiovascular. En J. Pla Vidal y J. Salvador Rodríguez (Coords.): Aspectos psicológicos en las enfermedades cardiovasculares. Madrid: Acción Médica.

Arthur, H.M., Patterson, C. y Stone, J.A. (2005). The role of complementary and alternative therapies in cardiac rehabilitation: a systematic evaluation. The European Journal of Cardiovascular Prevention and Rehabilitation, 13, 3-9.

Baer, R.A., Smith, G.T., Hopkins, J., Krietemeyer, J. y Toney, L. (2006). Using self-report assessment methods to explore facets of mindfulness. Assessment, 13(1), 27-45.

Balady, G.J., Williams, M.A., Ades, P.A., Bittner, V., Comoss, P., Foody, J.M. y American Association of Cardiovascular and Pulmonary Rehabilitation (2007). Core components of cardiac rehabilitation/secondary prevention programs: 2007 update: A scientific statement from the American Heart Association Exercise, Cardiac Rehabilitation, and Prevention Committee; the Council on Clinical Cardiology; the Councils on Cardiovascular Nursing, Epidemiology and Prevention, and Nutrition, Physical Activity, and Metabolism; and the American Association of Cardiovascular and Pulmonary Rehabilitation. Circulation, 115(20), 2675-2682.

Barnes, S., Brown, K.W., Krusemark, E., Campbell, W.K. y Rogge, R.D. (2007). The role of mindfulness in romantic relationship satisfaction and responses to relationship stress. Journal of Marital and Family Therapy, 33, 482-500.

Barrett, L.E. y Gross, J.J. (2001). Emotion representation and regulation: A process model of emotional intelligence. En T. Mayne y G. Bonnano (Eds.): Emotion: Current issues and future directions (pp. 286-310). New York: Guilford.

Blom, K., Baker, B., How, M., Dai, M., Irvine, J., Abbey, S. y Tobe, S.W. (2014). Hypertension analysis of stress reduction using mindfulness meditation and yoga: results from the HARMONY randomized controlled trial. American Journal of Hypertension, 27(1), 122-129. doi: 10.1093/ajh/hpt134.

Blumenthal, J.A., Sherwood, A. y Babyak, M.A. (2005). Effects of exercise and stress management training on markers of cardiovascular risk in patients with ischemic heart disease. A randomized controlled trial. Journal of the American Medical Association, 293(13), 1626-1634. doi: 10.1001/jama.293.13.1626.

Bohlmeijer, E., Prenger, R., Taal, E. y Cuijpers, P. (2010). The effects of mindfulness-based stress reduction therapy on mental health of adults with a chronic medical disease: a meta-analysis. Journal of Psychosomatic Research, 68, 539-544.

Bostock, S., Hamer, M., Wawrzyniak, A.J., Mitchell, E.S. y Steptoe, A. (2011). Positive emotional style and subjective, cardiovascular and cortisol responses to acute laboratory stress. Psychoneuroendocrinology, 36(8), 1175-1183. doi: 10.1016/j. psyneuen.2011.02.009.

Brewer, J.A., Mallik, S., Babuscio, T.A., Nich, C., Johnson, H.E., Deleone, C.M. y Rounsaville, B.J. (2011). Mindfulness training for smoking cessation: results from a randomized controlled trial. Drug and Alcohol Dependence, 119(1-2), 72-80.

Brown, K.W. y Cordon, S. (2009). Toward a phenomenology of mindfulness: subjective experience and emotional correlates. En F. Didonna (Ed.): Clinical handbook of mindfulness (pp. 59-81). New York: Springer.

Brown, K.W. y Ryan, R.M. (2003). The benefits of being present: Mindfulness and its role in psychological well-being. Journal of Personality and Social Psychology, 84(4), 822-848. 
Brown, K.W., Ryan, R.M. y Creswell, J.D. (2007). Mindfulness: Theoretical foundations and evidence for its salutary effects. Psychological Inquiry, 18(4), 211-237.

Cardaciotto, L., Herbert, J.D., Forman, E.M., Moitra, E. y Farrow, V. (2008). The assessment of present-moment awareness and acceptance: The Philadelphia Mindfulness Scale. Assessment, 15, 204-223.

Carney, R.M., Freedland, K.E., Miller, G.E. y Jaffe, A.S. (2002). Depression as a risk factor for cardiac mortality and morbidity. A review of potential mechanisms. Journal of Psychosomatic Research, 53, 897-902.

Castillo-Richmond, A., Schneider, R.H., Alexander, C.N., Cook, R., Myers, H., Nidich, S. y Salerno, J. (2000). Effects of stress reduction on carotid atherosclerosis in hypertensive African Americans. Stroke, 31, 568-573. [PubMed: 10700487].

Chambers, R., Lo, B.C. y Allen, N.B. (2008). The impact of intensive mindfulness training on attentional control, cognitive style, and affect. Cognitive Therapy and Research, 33, 302-322.

Chaves, G., Britez, N., Munzinger, J., Uhlmann, L., Gonzalez, G., Oviedo, G. y Mereles, D. (2015). Education to a healthy lifestyle improves symptoms and cardiovascular risk factors - AsuRiesgo Study. Arquivos Brasileiros de Cardiologia, 104(5), 347-355.

Chiesa, A. y Serretti, A. (2009). Mindfulness-based stress reduction for stress management in healthy people: a review and meta-analysis. Journal of Alternative and Complementary Medicine, 15, 593-600.

Chiesa, A. y Serretti, A. (2010). A systematic review of neurobiological and clinical features of mindfulness meditations. Psychological Medicine, 40, 1239-1252.

Clouse, R.E., Freedland, K.E., Carney, R.M., Griffith, P. y Lustman, P.J. (2001). Depression accelerates the presentation of coronary heart disease in women with diabetes mellitus. Psychosomatic Medicine, 63, 103.

Cohen, H.W., Gibson, G. y Alderman, M.H. (2000). Excess risk of myocardial infarction in patients treated with antidepressant medications: association of use of tricyclic agents. The American Journal of Medicine, 108, 2-8.

Creswell, J.D., Way, B.M., Eisenberger, N.I. y Lieberman, M.D. (2007). Neural correlates of mindfulness during affect labeling. Psychosomatic Medicine, 69, 560-565.

Cully, J.A., Stanley, M.A., Deswal, A., Hanania, N.A., Phillips, L.L. y Kunik, M.E. (2010). Cognitive-behavioral therapy for chronic cardiopulmonary conditions: preliminary outcomes from an open trial. Primary Care Companion to The Journal of Clinical Psychiatry, 12(4), e1-e6. Disponible en http://doi.org/10.4088/PCC.09m00896blu.

Davis, J.M., Goldberg, S.B., Anderson, M.C., Manley, A.R., Smith, S.S. y Baker, T.B. (2014). Randomized trial on mindfulness training for smokers targeted to a disadvantaged population. Substance Use and Misuse, 49(5), 571-585.

Day, W. (2000). Relaxation: a nursing therapy to help relieve cardiac chest pain. Australian Journal of Advanced Nursing, 18(1), 40-44.

De la Fuente, M., Franco, C. y Salvador, M. (2010). Reduction of blood pressure in a group of hypertensive teachers through a program of mindfulness meditation. Behavioral Psychology, 18, 533-552.

De Souza, I.C., De Barros, V.V., Gomide, H.P., Miranda, T.C., Menezes, V. de P., Kozasa, E.H. y Noto, A.R. (2015). Mindfulness-based interventions for the treatment of smoking: a systematic literature review. Journal of Alternative and Complementary Medicine, 21(3), 129-140. doi: 10. 1089/acm.2013.0471.

Dehdari, T., Heidarnia, A., Ramezankhani, A., Sadeghian, S. y Ghofranipour, F. (2009). Effects of progressive muscular relaxation training on quality of life in anxious patients after coronary artery bypass graft surgery. Indian Journal of Medical Research, 129(5), 603-608.

Dekker, R.L. (2011). Cognitive therapy for depression in patients with heart failure: a critical review. Heart Failure Clinics, 7(1), 127-141.

Delui, M.H., Yari, M., Khouyinezhad, G., Amini, M. y Bayazi, M.H. (2013). Comparison of cardiac rehabilitation programs combined with relaxation and meditation techniques on reduction of depression and anxiety of cardiovascular patients. The Open Cardiovascular Medicine Journal, 7, 99-103.

DiMatteo, M.R., Lepper, H.S. y Croghan, T.W. (2000). Depression is a risk factor for noncompliance with medical treatment. Archives of Internal Medicine, 160, 2101-2107.

Dimsdale, J.E. (2008). Psychological stress and cardiovascular disease. Journal of the American College of Cardiology, 51, $1237-1246$.

Drewnowski, A. (2009). Obesity, diets, and social inequalities. Nutrition Reviews, 67, S36-S39.

Durham, N.C. (2001). Depression and FCC. Archives of Internal Medicine. Aug. Disponible en http://www.news.mc.duke.edu.

Eaker, E.D., Sullivan, I.M., Kelly-Hayes, M., D’Agostino, R.B.S. y Benjamin, E.J. (2005). Tension and anxiety and the prediction of the 10-year incidence of coronary heart disease, atrial fibrillation, and total mortality: the Framingham Offspring Study. Psychosomatic Medicine, 67(5), 692-696.

Edelman, D., Oddone, E.Z., Liebowitz, R.S., Yancy, W.S.Jr., Olsen, M.K., Jeffreys, A.S. y Gaudet, T.W. (2006). A multidimensional integrative medicine intervention to improve cardiovascular risk. Journal of General Internal Medicine, 21(7), $728-734$. 
Edmondson, D., Green, P., Ye, S., Halazun, H.J. y Davidson, K.W. (2014). Psychological stress and 30-day all-cause hospital readmission in acute coronary syndrome patients: an observational cohort study. PLOS ONE, 9(3), e91477. doi: 10.1371/ journal.pone.0091477.

Elwafi, H.M., Witkiewitz, K., Mallik, S., Iv, T.A. y Brewer, J.A. (2013). Mindfulness training for smoking cessation: moderation of the relationship between craving and cigarette use. Drug and Alcohol Dependence, 130(1-3), 222-229.

Emani, S. y Binkley, P.F. (2010). Mind-body medicine in chronic heart failure. A translational science challenge. Circulation: Heart Failure, 3, 715-725.

Feldner, M.T., Zvolensky, M.J., Eifert, G.H. y Spira, A.P. (2003). Emotional avoidance: an experimental test of individual differences and response suppression using biological challenge. Behaviour Research and Therapy, 41, 403-411.

Feldman, G., Hayes, A., Kumar, S., Greeson, J. y Laurenceau, J. (2007). Mindfulness and emotion regulation: The development and initial validation of the Cognitive and Affective Mindfulness Scale-Revised (CAMS-R). Journal of Psychopathology and Behavioral Assessment, 29, 177-190.

Fortune, D.G., Richards, H.L., Main, C.J. y Griffiths, C.E.M. (2002). Patients' strategies for coping with psoriasis. Clinical and Experimental Dermatology, 27(3), 177-184.

Frewen, P.A., Evans, E.M., Maraj, N., Dozois, D.J. y Partridge, K. (2008). Letting go: Mindfulness and negative automatic thinking. Cognitive Therapy and Research, 32, 758-774.

García-Gómez, R.G., Lopez-Jaramillo, P. y Tomaz, C. (2007). The role played by the autonomic nervous system in the relation between depression and cardiovascular disease. Revista de Neurología, 44(4), 225-233.

Giallauria, F., Lucci, R., D’Agostino, M., Vitelli, A., Maresca, L., Mancini, M. y Vigorito, C. (2009). Two-year multicomprehensive secondary prevention program: favorable effects on cardiovascular functional capacity and coronary risk profile after acute myocardial infarction. Journal of Cardiovascular Medicine, 10(10), 772-780.

Gross, J.J. y Thompson, R.A. (2007). Emotion regulation: Conceptual foundations. En J.J. Gross (Ed.): Handbook of emotion and regulation (pp. 3-24). New York: Guilford.

Hamer, M., Molloy, G.J. y Stamatakis, E. (2008). Psychological distress as a risk factor for cardiovascular events: pathophysiological and behavioral mechanisms. Journal of the American College of Cardiology, 52, 2156-2162.

Hartmann, M., Kopf, S., Kircher, C., Faude-Lang, V., Djuric, Z., Augstein, F. y Nawroth, P.P. (2012). Sustained effects of a mindfulness-based stress-reduction intervention in type 2 diabetic patients: design and first results of a randomized controlled trial (the Heidelberger Diabetes and Stress-Study). Diabetes Care, 35(5), 945-947.

Hayes, A.M. y Feldman, G. (2004). Clarifying the construct of mindfulness in the context of emotion regulation and the process of change in therapy. Clinical Psychology: Science and Practice, 11, 255-262.

Hayes, S.C., Strosahl, K. y Wilson, K.G. (1999). Acceptance and commitment therapy: An experiential approach to behavior change. New York: The Guilford Press.

Hayman, L.L., Williams, C.L., Daniels, S.R., Steinberger, J., Paridon, S., Dennison, B.A. y McCrindle, B.W. (2004). Cardiovascular Health Promotion in the Schools. A statement for health and education professionals and child health advocates from the Committee on Atherosclerosis, Hypertension, and Obesity in Youth (AHOY) of the Council on Cardiovascular Disease in the Young, American Heart Association. Circulation, 110, 2266-2275.

Hervás, G., Cebolla, A. y Soler, J. (2016). Intervenciones psicológicas basadas en mindfulness y sus beneficios: estado actual de la cuestión. Clínica y Salud, 27, 115-124.

Hodgins, H.S. y Knee, C.R. (2002). The integrating self and conscious experience. En E. L. Deci, y R. M. Ryan (Eds.): Handbook of Self-Determination Research (pp. 87-100). New York: University of Rochester Press.

Hughes, J.W., Fresco, D.M., Myerscough, R., van Dulmen, H.M., Carlson. L.E. y Josephson, R. (2013). Randomized controlled trial of mindfulness-based stress reduction for prehypertension. Psychosomatic Medicine, 75, 721-728.

Instituto Nacional de Salud y Observatorio Nacional de Salud (2014). "Enfermedad cardiovascular". En Tercer Informe ONS: Mortalidad evitable en Colombia para 1998-2011 (p. 163). Bogotá: Imprenta Nacional de Colombia. Recuperado de http:// www.ins.gov.co/lineas-de-accion/ons/SiteAssets/Paginas/publicaciones/Informe3-ME-ONS-Definitivo.pdf.

Ivanova, E., Jensen, D., Cassoff, J., Gu, F. y Knauper, B. (2015). Acceptance and commitment therapy improves exercise tolerance in sedentary women. Medicine \& Science in Sports and Exercise, 47(6), 1251-1258.

Jacobs, G.D. (2001). Clinical applications of the relaxation response and mind-body interventions. Journal of Alternative and Complementary Medicine, 7(Suppl. 1), 93-101.

Jerant, A., Moore, M., Lorig, K. y Franks, P. (2008). Perceived control moderated the self-efficacy-enhancing effects of a chronic illness selfmanagement intervention. Chronic Illness, 4(3), 173-182.

Johansson, B., Bjuhr, H. y Ronnback, L. (2012). Mindfulness-based stress reduction (MBSR) improves long-term mental fatigue after stroke or traumatic brain injury. Brain Injury, 26, 1621-1628.

Jolliffe, J.A., Rees, K., Taylor, R.S., Thompson, D., Oldridge, N. y Ebrahim, S. (2001). Exercise-based rehabilitation for coronary heart disease. Cochrane Database of Systematic Reviews, (1): CD001800. 
Joo, H.M., Lee, S.J., Chung, Y.G. y Shin, I.Y. (2010). Effects of mindfulness based stress reduction program on depression, anxiety and stress in patients with aneurysmal subarachnoid hemorrhage. Journal of Korean Neurosurgical Society, 47, 345-351.

Joynt, K.E., Whellan, D.J. y O'Connor, C.M. (2003). Depression and cardiovascular disease: mechanisms of interaction. Biological Psychiatry, 54(3), 248-261.

Kabat-Zinn, J. (2003). Mindfulness-based interventions in context: Past, present, and future. Clinical Psychology: Science and Practice, 10, 144-156.

Kabat-Zinn, J. (2013). Full catastrophe living: using the wisdom of your body and mind to face stress, pain, and illness. New York: Bantam.

Kabat-Zinn, J., Lipworth, L. y Burney, R. (1985). The clinical use of mindfulness meditation for the selfregulation of chronic pain. Journal of Behavioral Medicine, 8(2), 163-190.

Kabat-Zinn, J., Massion, A.O., Kristeller, J., Peterson, L.G., Fletcher, K.E., Pbert, L. y Santorelli, S.F. (1992). Effectiveness of a meditation based stress reduction program in the treatment of anxiety disorders. American Journal of Psychiatry, 149, 936-943.

Kangasniemi, A.M., Lappalainen, R., Kankaanpaa, A., Tolvanen, A. y Tammelin, T. (2015). Towards a physically more active lifestyle based on one's own values: The results of a randomized controlled trial among physically inactive adults. $B M C$ Public Health, 15, 260.

Kearney, D.J., Milton, M.L., Malte, C.A., McDermott, K.A., Martinez, M. y Simpson, T.L. (2012). Participation in mindfulness-based stress reduction is not associated with reductions in emotional eating or uncontrolled eating. Nutrition Research, 32(6), 413-420.

Keen, A.J.A., Duncan, E. y Gold, A.E. (2012). The effectiveness of a mindfulness-based cognitive therapy intervention specifically designed for adults with diabetes experiencing significant levels of anxiety and/or depression: a pilot clinical service. Diabetic Medicine, 29, 161.

Kelly, J.F. y Greene, M.C. (2014). Where there's a will there's a way: a longitudinal investigation of the interplay between recovery motivation and self-efficacy in predicting treatment outcome. Psychology of Addictive Behaviors, 28(3), 928-934. doi: $10.1037 / \mathrm{a} 0034727$.

Keng, S.L., Smoski, M.J. y Robins, C.J. (2011). Effects of mindfulness on psychological health: A review of empirical studies. Clinical Psychology Review, 31(6), 1041-1056.

Khawaja, I.S., Westermeyer, J.J., Gajwani, P. y Feinstein, R.E. (2009). Depression and coronary artery disease: the association, mechanisms, and therapeutic implications. Psychiatry (Edgmont), 6(1), 38-51.

Kiken, L. y Shook, N. (2011). Looking up: Mindfulness increases positive judgments and reduces negativity bias. Social Psychology \& Personality Science, 2, 425-431.

Kimberly, Y., Forrest, Z., Becker, D.J., Kuller, L.H., Wolfson, S.K. y Orchard, T.J. (2000). Are predictors of coronary heart disease and lower extremity arterial disease in type 1 diabetes the same? Atherosclerosis, 148, 159-169.

Kreitzer, M.J. y Snyder, M. (2002). Healing the hearth: Integrating complementary therapies and healing practices into the care of cardiovascular patients. Progress in Cardiovascular Nursing, 17, 73-80.

Kubzansky, L.D. y Kawachi, I. (2000). Going to the heart of the matter: Do negative emotions cause coronary heart disease? Journal of Psychosomatic Research, 48(4-5), 323-337.

Lampinen, P., Heikkinen, R.L. y Ruoppila, I. (2000). Changes in intensity of physical exercise as predictors of depressive symptoms among older adults: an eight-year follow-up. Preventive Medicine, 30(5), 371-380.

Lane, D., Carroll, D., Ring, C., Beevers, G., y Lip, G. (2001). Mortality and quality of life 12 months after myocardial infarction: effects of depression and anxiety. Psychosomatic Medicine, 63, 221-230.

Langer, A.I., Ulloa, V.G., Cangas, A.J., Rojas, G. y Krause, M. (2015). Mindfulness-based interventions in secondary education: a qualitative systematic review. Studies in Psychology, 36(3), 533-570. doi: http://dx.doi.org/10.1080/02109395.2015.10 78553

Larsen, R. (2000). Toward a science of mood regulation. Psychological Inquiry, 11(3), 129-141. doi: 10.1207/S15327965PLI1103 01.

Lawrence, M., Booth, J., Mercer, S. y Crawford, E. (2013). A systematic review of the benefits of mindfulness-based interventions following transient ischemic attack and stroke. International Journal of Stroke, 8, 465-474.

Leonaite, A. y Vainoras, A. (2010). Heart rate variability during two relaxation techniques in post-MI men. Electronics and Electrical Engineering, 5(101), 107-110.

Levitt, J.T., Brown, T.A., Orsillo, S.M. y Barlow, D.H. (2004). The effects of acceptance versus suppression of emotion on subjective and psychophysiological response to carbon dioxide challenge in patients with panic disorder. Behavior Therapy, $35,747-766$.

Linehan, M. (1993). Cognitive behavioural treatment for borderline personality disorder. New York: The Guilford Press. 
Intervenciones basadas en mindfulness para reducir el estrés en la enfermedad cardiovascular: ¿cómo funcionan?

Linehan, M.M., Cochran, B.N. y Kehrer, C.A. (2001). Dialectical behavior therapy for borderline personality disorder. En D.H. Barlow (Ed.): Clinical handbook of psychological disorders: A step-by-step treatment manual (3rd ed.) (p. 470-522). New York: The Guilford Press.

Loucks, E.B., Britton, W.B., Howe, C.J., Eaton, C.B. y Buka, S.L. (2015). Positive associations of dispositional mindfulness with cardiovascular health: the New England Family Study. International Journal of Behavioral Medicine, 22(4), 540-550.

Loucks, E.B., Schuman-Oliver, Z., Britton, W.B., Fresco, D.M., Desbordes, G., Brewer, J.A. y Fulwiler, C. (2015). Mindfulness and cardiovascular disease risk: State of the evidence, plausible mechanisms, and theoretical framework. Current Cardiology Reports, 17, 112.

Ludwing, D.S. y Kabat-Zinn, J. (2008). Mindfulness in Medicine. Journal of the American Medical Association, 300(11), 1350-1352. doi: 10.1001/jama.300.11.1350.

Lyne, K. y Roger, D. (2000). A psychometric re-assessment of the COPE questionnaire. Personality and Individual Differences, 29(2), 321-335.

Manchada, S.C. y Madan, K. (2014). Yoga and meditation in cardiovascular disease. Clinical Research in Cardiology, 103(9), 675-680. doi: 10.1007/s00392-014-0663-9.

Marlatt, G.A. y Donovan D.M. (2005). Relapse prevention: maintenance strategies in the treatment of addictive behaviors. New York: The Guilford Press.

Mars, T.S. y Abbey, H. (2010). Mindfulness meditation practise as a healthcare intervention: a systematic review. International Journal of Osteopathic Medicine, 13, 56-66.

McEwen, B. (2000). Allostasis and allostatic load: implications for neuropsy-chopharmacology. Neuropsychopharmacology, 22, 108-124.

McKee, L., Zvolensky, M.J., Solomon, S.E., Bernstein, A. y Leen-Feldner, E. (2007). Emotional-vulnerability and mindfulness: A preliminary test of associations among negative affectivity, anxiety sensitivity, and mindfulness skills. Cognitive Behaviour Therapy, 36, 91-100.

Mehling, W.E., Gopisetty, V., Daubenmier, J., Price, C.J., Hecht, F.M. y Stewart, A. (2009). Body awareness: construct and self-report measures. PLOS ONE, 4(5), e5614.

Messner, B. y Bernhard, D. (2014). Smoking and cardiovascular disease. mechanisms of endothelial dysfunction and early atherogenesis. Arteriosclerosis, Thrombosis, and Vascular Biology, 34, 509-515.

Miller, C.K., Kristeller, J.L., Headings, A. y Nagaraja, H. (2014). Comparison of a mindful eating intervention to a diabetes self-management intervention among adults with type 2 diabetes: a randomized controlled trial. Health Education and Behavior, 41, 145-154.

Ministerio de Salud y Protección Social (21 de marzo de 2014). “Colombia enfrenta epidemia de enfermedades cardiovasculares $y$ diabetes”. Boletín de Prensa $\mathrm{N}^{\circ} 077$. Boogotá: MSPS.

Miró, M.T., Perestelo-Pérez, L., Pérez, J., Rivero, A., González, M., De la Fuente, J. y Serrano, P. (2011). Eficacia de los tratamientos basados en mindfulness para los trastornos de ansiedad y depression: Una revision sistemática. Revista de Psicopatología y Psicología Clínica, 16(1), 1-14.

Moffitt, R. y Mohr, P. (2015). The efficacy of a self-managed acceptance and commitment therapy intervention DVD for physical activity initiation. British Journal of Health Psychology, 20(1), 115-129.

Moustgaard, A., Bedard, M. y Felteau, M. (2007). Mindfulness-based cognitive therapy (MBCT) for individuals who had a stroke: results from a pilot study. Journal of Cognitive Rehabilitation, 25, 4-10.

Musselman, D.L., Marzec, U.M., Manatunga, A., Penna, S., Reemsnyder, A., Knight, B.T. y Nemeroff, C.B. (2000). Platelet reactivity in depressed patients treated with paroxetine: preliminary findings. Archives of General Psychiatry, 57, 875-882.

Naranjo E., N.F., Díaz Q., F.A. y García, R.G. (2012). The influence of cardiac rehabilitation on acute myocardial infarction patients' readmission rate in Santander, Colombia. Revista de Salud Pública, 14(5), 831-841.

Neves, A., Alves, A. J., Ribeiro, F., Lopes G., J. y Oliveira, J. (2009). The effect of cardiac rehabilitation with relaxation therapy on psychological, hemodynamic, and hospital admission outcome variables. Journal of Cardiopulmonary Rehabilitation and Prevention, 29, 304-309.

Nicholson, A., Kuper, H. y Hemingway, H. (2006). Depression as an aetiologic and prognostic factor in coronary heart disease: a meta-analysis of 6362 events among 146,538 participants in 54 observational studies. European Heart Journal, 27, 27632774.

Nyklíček, I., Dijksman, S.C., Lenders, P.J, Fonteijn, W.A. y Koolen, J.J. (2014). A brief mindfulness based intervention for increase in emotional well-being and quality of life in percutaneous coronary intervention (PCI) patients: The Mindful Heart randomized controlled trial. Journal of Behavioral Medicine, 37(1), 135-144.

Olson, K.L. y Emery, C.F. (2015). Mindfulness and weight loss: a systematic review. Psychosomatic Medicine, 77(1), 59-67.

O’Reilly, G.A., Cook, L., Spruijt-Metz, D. y Black, D.S. (2014). Mindfulness based interventions for obesity-related eating behaviours: a literature review. Obesity Reviews, 15(6), 453-461. 
Organización Mundial de la Salud (2011). Informe sobre la situación mundial de las enfermedades no transmisibles 2010. Resumen de orientación. Recuperado de http://www.who.int/nmh/publications/ncd_report_summary_es.pdf

Organización Mundial de la Salud (2015, enero). “Enfermedades cardiovasculares”. Nota Descriptiva. Ginebra: OMS. Recuperado de http://www.who.int/mediacentre/factsheets/fs317/es/.

Ouwens, M.A., Schiffer, A.A., Visser, L.I., Raeijmaekers, N.J. y Nyklicek, I. (2015). Mindfulness and eating behaviour styles in morbidly obese males and females. Appetite, 87, 62-67.

Pace, T.W.W., Negi, L.T., Adame, D.D., Cole, S.P., Sivilli, T.I., Brown, T.D. y Raison, C.L. (2009). Effect of compassion meditation on neuroendocrine, innate immune and behavioral responses to psychosocial stress. Psychoneuroendocrinology, 34(1), 87-98.

Papaioannou, V., Pneumatikos, I. y Maglaveras, N. (2013). Association of heart rate variability and inflammatory response in patients with cardiovascular diseases: current strengths and limitations. Frontiers in Physiology, 4(174). Recuperado de http://doi.org/10.3389/fphys.2013.00174.

Phillips, J.E. y Klein, W.M.P. (2010). Socioeconomic status and coronary heart disease risk: the role of social cognitive factors. Social and Personality Psychology Compass, 4(9), 704-727. doi: 10.1111/j.1751-9004.2010.00295.x.

Pollock, B.G., Laghrissi-Thode, F. y Wagner, W.R. (2000). Evaluation of platelet activation in depressed patients with ischemic heart disease after paroxetine or nortriptyline treatment. Journal of Clinical Psychopharmacology, 20(2), 137-140.

Power, C., Hypponen, E. y Smith, G.D., (2005). Socioeconomic position in childhood and early adult life and risk of mortality: a prospective study of the mothers of the 1958 British birth cohort. American Journal of Public Health, 95, $1396-1402$.

Proulx, K. (2008). Experiences of women with bulimia nervosa in a mindfulness based eating disorder treatment group. Eating Disorders, 16(1), 52-72.

Reibel, D.K., Greeson, J.M., Brainard, G.C. y Rosenzweig, S. (2001). Mindfulness-based stress reduction and health-related quality of life in a heterogeneous patient population. General Hospital Psychiatry, 23, 183-192.

Richardson, S., Shaffer, J.A., Falzon, L., Krupka, D., Davidson, K.W. y Edmondson, D. (2012). Meta-analysis of perceived stress and its association with incident coronary heart disease. American Journal of Cardiology, 110, 1711-1716.

Roest, A.M., Martens, E.J., de Jonge, P. y Denoller, J. (2010). Anxiety and risk of incident coronary heart disease: a meta-analysis. Journal of the American College of Cardiology, 56, 38-46.

Roest, A.M., Martens, E.J., Denollet, J. y de Jonge, P. (2010). Prognostic association of anxiety post myocardial infarction with mortality and new cardiac events: a meta-analysis. Psychosomatic Medicine, 72, 563-569.

Rosenzweig, S., Reibel, D.K., Greeson, J.M., Edman, J.S., Jasser, S.A., McMearty, K.D. y Goldstein, B.J. (2007). Mindfulness-based stress reduction is associated with improved glycemic control in type 2 diabetes mellitus: a pilot study. Alternative Therapies in Health and Medicine, 13, 36-38.

Ruiz, M. (2003). Factores de riesgo cardiovascular en niños y adolescentes. Madrid: Díaz Santos.

Ryan, R.M. y Deci, E.L. (2000). Self-determination theory and the facilitation of intrinsic motivation, social development, and well-being. American Psychology, 55, 68-78.

Salmoirago-Blotcher, E., Husinger, M., Morgan, L., Fischer, D. y Carmody, J. (2013). Mindfulness-based stress reduction and change in health-related behaviors. Journal of Evidence-Based Complementary \& Alternative Medicine, $18(4), 243-247$.

Sánchez P., E.M. y Moreno C., A. (2017). Efectos del tratamiento de atención plena y compasión sobre ansiedad, calidad de vida y asma. Psicología y Salud, 27(2), 167-178.

Sandín, B. (2001). Papel de las emociones negativas en el trastorno cardiovascular: Un análisis crítico. Revista de Psicopatología y Psicología Clínica, 7(1), 1-18.

Santorelli, S. (Ed.) (2014). Mindfulness-Based Stress Reduction (MBSR): Standards of practice. Worcester, MA: University of Massachusetts Medical School.

Schneider, R.H., Grim, C.E., Rainforth, M.V., Kotchen, T., Sanford, I.N., Gaylord-King, C. y Alexander, C.N. (2012). Stress reduction in the secondary prevention of cardiovascular disease: Randomized, controlled trial of transcendental meditation and health education in blacks. Circulation: Cardiovascular Quality and Outcomes, 5, 750-758. doi: 10.1161/CIRCOUTCOMES.112.967406.

Segal, Z.V., Williams, J.M.G. y Teasdale, J.D. (2002). Mindfulness-based cognitive therapy for depression. NewYork: The Guilford Press.

Shapiro, S.L., Schwartz, G.E. y Bonner, G. (1998). Effects of mindfulness-based stress reduction on medical and premedical students. Journal of Behavioral Medicine, 21, 581-599.

Shapiro, S.L., Brown, K.W. y Biegel, G. (2007). Teaching self-care to caregivers: The effects of mindfulness-based stress reduction on the mental health of therapists in training. Training and Education in Professional Psychology, 1, $105-115$.

Shapiro, S.L., Carlson, L.E., Astin, J.A. y Freedman, B. (2006). Mechanisms of mindfulness. Journal of Clinical Psychology, $62(3), 373-386$.

Sheila, S., Irvin, B.L., Lin, H.S. y Mar, C.L. (2003). Effects of progressive muscle relaxation on blood pressure and psychosocial status for clients with essential hypertension in Taiwan. Holistic Nursing Practice, 17(1), 41-47. 
Sloan, D.M. (2004). Emotion regulation in action: Emotional reactivity in experiential avoidance. Behavior Research and Therapy, 42, 1257-1270.

Speca, M., Carlson, L.E., Goodey, E. y Angen, M. (2000). A randomized, wait-list controlled clinical trial: The effect of a mindfulness meditation based stress reduction program on mood and symptoms of stress in cancer outpatients. Psychosomatic Medicine, 62, 613-622.

Steptoe, A. y Kivimäki, M. (2013). Stress and cardiovascular disease: an update on current knowledge. Annual Review of Public Health, 34, 337-354.

Steptoe, A. y Kivimäki, M. (2012). Stress and cardiovascular disease. Nature Reviews Cardiology, 9(6), 360-370.

Steptoe, A., Shankar, A., Demakakos, P. y Wardle, J. (2013). Social isolation, loneliness, and all-cause mortality in older men and women. Proceedings of the National Academy of Sciences, 110(15), 5797-5801.

Strik, J., Denollet, J., Lousberg, R. y Honig, A. (2003). Comparing symptoms of depression and anxiety as predictors of cardiac events and increased health care consumption after myocardial infarction. Journal of the American College of Cardiology, 42(10), 1801-1807.

Stowell, J.R., Kiecolt-Glaser, J.K. y Glaser, R. (2001). Perceived stress and cellular immunity: When coping counts. Journal of Behavioral Medicine, 24(4), 323-339.

Sullivan, M.J., Wood, L., Terry, J., Brantley, J., Charles, A., McGee, V. y Cuffe, M.S. (2009). The Support, Education, and Research in Chronic Heart Failure Study (SEARCH): A mindfulness-based psychoeducational intervention improves depression and clinical symptoms in patients with chronic heart failure. American Heart Journal, 157, 84-90.

Tacón, A.M., Caldera, Y.M. y Ronaghan, C. (2004). Mindfulness-based stress reduction in women with breast cancer. Families, Systems \& Health, 22, 193-203.

Tak, S.R., Hendrieckx, C., Nefs, G., Nyklicek, I., Speight, J. y Pouwer, F. (2015). The association between types of eating behaviour and dispositional mindfulness in adults with diabetes. Results from Diabetes MILES. The Netherlands. Appetite, 87, 288-295.

Teasdale, J.D., Segal, Z.V., Williams, J.M., Ridgeway, V.A., Soulsby, J.M. y Lau, M.A. (2000). Prevention of relapse/recurrence in major depression by mindfulness-based cognitive therapy. Journal of Consulting and Clinical Psychology, 68(4), 615-623.

Thurston, R.C. y Kubzansky, L.D. (2009). Women, loneliness, and incident coronary heart disease. Psychosomatic Medicine, $71(8), 836-842$.

Thurston, R.C., Rewak, M. y Kubzansky, L.D. (2013). An anxious heart: anxiety and the onset of cardiovascular diseases. Progress in Cardiovascular Diseases, 55, 524-537.

Ulmer, C.S., Stetson, B.A. y Salmon, P.G. (2010). Mindfulness and acceptance are associated with exercise maintenance in YMCA exercisers. Behaviour Research and Therapy, 48(8), 805-809.

Vallejo, M.A. (2006). Mindfulness. Papeles del Psicólogo, 27(2), 92-99.

van Dixhoorn, J. y White, A. (2005). Relaxation therapy for rehabilitation and prevention in ischaemic heart disease: a systematic review and meta-analysis. European Journal of Cardiovascular Prevention and Rehabilitation, 12(3), $193-202$.

van Son, J., Nyklíček, I., Pop, V.J.M., Blonk, M., Erdtsieck, R., Spooren, P. y Pouwer, F. (2013). The effects of a mindfulness-based intervention on emotional distress, quality-of-life, and HbA1c in outpatients with diabetes (DiaMind). Diabetes Care, $13,823-830$.

Varela, E. y Depraz, N. (2003). Imagining: Embodiment, phenomenology, and transformation. En B.A. Wallace (Ed.): Buddhism and science: Breaking new ground (pp. 195-232). New York: Columbia University Press.

Varvogli, L. y Darviri, C. (2011). Stress management techniques: evidence-based procedures that reduce stress and promote health. Health Science Journal, 5(2), 74-89.

Vickers, A., Zollman, C. y Payne, D.K. (2001). Hypnosis and relaxation therapies. The Western Journal of Medicine, 175(4), 269-272.

Villablanca, A.C., McDonald, J.M. y Rutledge, J.C. (2000). Smoking and cardiovascular disease. Clinics in Chest Medicine, 21(1), 159-172.

Wachs, K. y Cordova, J.V. (2007). Mindful relating: Exploring mindfulness and emotion repertoires in intimate relationships. Journal of Marital \& Family, 33, 464-481.

Walton, K.G., Schneider, R.H. y Nidich, S.I. (2004). Review of controlled research on the transcendental meditation program and cardiovascular disease: risk factors, morbidity, and mortality. Cardiology in Review, 12(5), 262-266.

Walton, K.G., Schneider, R.H., Nidich, S.I., Salerno, J.W., Nordstrom, C.K. y Merz, C.N.B. (2002). Psychosocial stress and cardiovascular disease, Part 2: Effectiveness of the transcendental meditation program in treatment and prevention. Behavioral Medicine, 28(3), 106-123.

Wansink, B., Painter, J.E. y North, J. (2005). Bottomless bowls: why visual cues of portion sizes may influence intake. Obesity Research, 13(1), 93-100. 
Williams, K.A., Kolar, M.M., Reger, B.E. y Pearson, J.C. (2001). Evaluation of a wellness-based mindfulness stress reduction intervention: A controlled trial. American Journal of Health Promotion, 15, 422-432.

Witte, D.R., Bots, M.L., Hoes, A.W. y Grobbee, D.E. (2000). Cardiovascular mortality in Dutch men during 1996 European football championship: longitudinal population study. British Medical Journal, 321, 1552-1554.

Wupperman, P., Neumann, C.S. y Axelrod, S.R. (2008). Do deficits in mindfulness underlie borderline personality features and core difficulties? Journal of Personality Disorders, 22(5), 466-482.

Yeh, G.Y., Davis, R.B. y Phillips, R.S. (2006). Use of complementary therapies in patients with cardiovascular disease. American Journal of Cardiology, 98(5), 673-680.

Yeung, S. (2013). Mindfulness, negative cognition and mental health. SS Student E-Journal, 2, 49-67.

Younge, J.O., Gotink, R.A., Baena, C.P., Roos-Hesselink, J.W. y Hunink, M. (2014). Mind-body practices for patients with cardiac disease: a systematic review and meta-analysis. European Journal of Preventive Cardiology, 22(11), 1385-1398. doi: $10.1177 / 2047487314549927$.

Ziegelstein, R.C., Fauerbach, J.A., Stevens, S.S., Romanelli, J., Richter, D.P. y Bush, D.E. (2000). Patients with depression are less likely to follow recommendations to reduce cardiac risk during recovery from a myocardial infarction. Archives of Internal Medicine, 160, 1818-1823. 